\title{
Entropy and the second law for driven, or quenched, thermally isolated systems
}

\author{
Udo Seifert
}

II. Institut für Theoretische Physik, Universität Stuttgart, 70550 Stuttgart, Germany

\begin{abstract}
The entropy of a thermally isolated system should not decrease after a quench or external driving. For a classical system following Hamiltonian dynamics, we show how this statement emerges for a large system in the sense that the extensive part of the entropy change does not become negative. However, for any finite system and small driving, the mean entropy change can well be negative. We derive these results using as micro-canonical entropy a variant recently introduced by Swendsen and co-workers called "canonical". This canonical entropy is the one of a canonical ensemble with the corresponding mean energy. As we show by refining the micro-canonical Crooks relation, the same results hold true for the two more conventional choices of micro-canonical entropy given either by the area of a constant energy shell, the Boltzmann entropy, or the volume underneath it, the Gibbs volume entropy. These results are exemplified with quenched $N$-dimensional harmonic oscillators.
\end{abstract}

Keywords: entropy, second law, micro-canonical Crooks relation

\section{Introduction}

A main purpose of the concept of entropy is to rationalize the second law. In one of its incarnations it says that in a driven isolated system, entropy should not decrease. This scenario includes the more restricted cases of releasing a constraint or establishing contact between previously separated systems, since both processes can be realized by a sudden change of a control parameter in the total Hamiltonian, i.e., by a quench. Even if one accepts the crucial assumption that an isolated system finally equilibrates, as we will do throughout this paper, there remains the task to show that the entropy does not decrease.

The quest for a proof of such a dynamical version of the second law involving entropy should conceptually be distinguished from apparently related results for second law-like statements involving the mean work spent or extracted in such processes for which one must distinguish two types of initial conditions. For sampling from a canonical distribution, one can prove the Kelvin-Planck statement of the second law asserting that work cannot be extracted from a cyclic variation even for a finite system [1, 2, 3, 4]. On the other hand, for micro-canonical initial conditions, there are explicit examples of low-dimensional systems that demonstrate the contrary [5, 6]. Crucially for the present context, these, and related [7] results about work do not require, or invoke, any notion of entropy and hence do not imply a second law for entropy without additional assumptions.

Various forms for the entropy for an isolated system in equilibrium have recently been discussed, compared and criticised, see [8, 9] for review-like presentations. One standard candidate for entropy under micro-canonical initial conditions is the one derived from the size of a thin energy shell, often called Boltzmann entropy. One of its obvious short-comings is the formal necessity to introduce an ill-defined thickness of the energy shell to get the dimensions correct. A complementary definition often called Gibbs volume entropy that involves all states below a certain energy avoids this fuzziness. Recently, introducing a third variant, Swendsen and co-workers have suggested to assign to an isolated quantum system the standard entropy of that canonical ensemble that has as mean energy the originally given micro-canonical energy [10, 11]. We will adapt this definition to classical systems with unbounded kinetic energy

Email address: useifert@theo2.physik.uni-stuttgart.de (Udo Seifert) 
that are of interest here. While for a large system with a monotonically increasing density of states, all three variants become equivalent, they differ in the extent to which they obey, for finite systems or systems with a non-monotonic density of states, the various axioms of thermodynamics. Quoting recent representative work emphasizing the merits of the Boltzmann entropy [12, 13, 14], Gibbs volume entropy [15, 16], canonical entropy [10, 11] and still another one [17] should suffice to get an entry to this debate, which is not the main topic of this work.

The main purpose of the present paper is rather to explore the potential of the three variants, and, in particular, that of canonical entropy as the least well-known for deriving a second law for driven systems from a dynamical perspective. Assuming equilibration in an isolated system and Hamiltonian dynamics, we first prove that the canonical entropy change evaluated at the canonical average of the final energy is non-negative for a finite system and microcanonical initial conditions. With minor additional assumptions about the scaling of the cumulants of work for large systems, this theorem shows how a second law for the canonical entropy change of a large system emerges in the sense that, for a large system, an extensive negative entropy change can be shown not to exist. However, for small driving the mean entropy change can well become negative for any fixed system size. Second, starting from the micro-canonical Crooks relation [18] and refining it, we explore the emergence of a second law for large systems also explicitly for the Boltzmann and the Gibbs entropy.

For the present crucial micro-canonical initial conditions, earlier work in this direction includes the observation that for quenched one-dimensional non-linear oscillators the Gibbs volume entropy can decrease [19, 20]. For large systems, Sasa and Komatsu show first that for a small quench the change in volume entropy is not extensively negative. In a second step, using concepts from chaotic dynamics, they conclude the same property for an arbitrary (most probable) process [21]. Tasaki proves an increase in volume entropy in the large $N$-limit [22]. For quenching or driving from canonical initial conditions, an increase in entropy can be shown even for a finite system using the Gibbs volume entropy [22, 23].

It should be emphasized that the present approach and the results derived below are not in conflict with the wellknown fact that under Hamiltonian dynamics a fine-grained non-equilibrium entropy of the form $-\int d \boldsymbol{\xi} p(\boldsymbol{\xi}, t) \ln p(\boldsymbol{\xi}, t)$ is conserved due to Liouville's theorem. Starting with such a fine-grained entropy, second law-like statements can still be obtained, either by a final weak coupling to an ideal bath [24], or by breaking dynamically induced correlations between system and bath [25]. Even for a system strongly coupled to a heat bath, a second law can then be proven for the sum of a suitably identified system entropy change and the entropy change of the bath due to the exchanged heat [26], see also [27, 28, 29, 30]. In the present paper, we deal with a more coarse-grained entropy function that depends only on energy and the control parameters and that can be defined as an equilibrium property. As is well-known, demonstrating explicitly a final equilibration after a quench or driving is a quite different, much more difficult issue not addressed here.

The paper is organized as follows. In Sect. II, we state the problem. In Sect. III, we recall the definition of canonical entropy and derive the corresponding second law. In Sect. IV, starting with the micro-canonical Crooks relation, we analyze the status of a dynamical second law for the two standard variants of micro-canonical entropy. In Sect. V, two variants of quenched harmonic oscillators are presented as specific examples. We conclude in Sect. VI.

\section{The problem}

We consider an isolated system characterized by a $N$-particle Hamiltonian $H(\xi, \lambda)$ that depends on the degrees of freedom $\xi$ and a control parameters $\lambda$ through which we drive the system for a finite time $0 \leq \tau \leq t$ from $\lambda^{0}$ to $\lambda^{1}$ leading to a trajectory $\xi^{\tau}$ in phase space. The phase point at the final time $t$ becomes a function of the initial one, $\xi^{1}\left(\xi^{0}\right) \equiv \xi^{t}\left(\xi^{0}\right)$. The work

$$
W\left(\xi^{0}\right) \equiv H\left(\xi^{1}\left(\xi^{0}\right), \lambda^{1}\right)-E^{0}
$$

spent in this process is the total energy change with $E^{0}=H\left(\xi^{0}, \lambda^{0}\right)$. A necessary condition for a second law to hold under this driving is that there exists an entropy $S(E, \lambda)$ such that

$$
\left.\left\langle S\left(E^{0}+W\left(\xi^{0}\right)\right), \lambda^{1}\right)\right\rangle-S\left(E^{0}, \lambda^{0}\right) \geq 0
$$

for any arbitrary but fixed driving protocol $\lambda^{\tau}$. Throughout, averages $\langle\ldots\rangle$ are over micro-canonical initial conditions, i.e., over the initial energy shell with energy $E^{0}$.

In the following, we explore the status of such a putative second law both for finite systems and in the thermodynamic limit using three version of entropy. 


\section{Second law for canonical entropy}

\subsection{Definition of canonical entropy}

For the isolated system with energy $E$ and a Hamiltonian $H(\xi, \lambda)$, "canonical" entropy $S_{C}(E, \lambda)$ is defined through considering a fictitious canonical ensemble at a temperature that would lead to a mean energy $E$ [10]. Specifically, this substitute, or "canonical", (inverse) temperature $\beta_{C}(E, \lambda)$ follows from solving the implicit equation

$$
E=-\partial_{\beta} \ln Z(\beta, \lambda)_{\beta=\beta_{C}(E, \lambda)}
$$

for $\beta_{C}(E, \lambda)$. Here, the canonical partition function is given by the usual

$$
Z(\beta, \lambda) \equiv \int d \xi \exp [-\beta H(\xi, \lambda)] \equiv \exp [-\beta F(\beta, \lambda)],
$$

with the free energy $F(\beta, \lambda)$. The integration is over all phase space with normalization factors like Planck's constant and $N$ factorials notationally suppressed. Once $\beta_{C}(E, \lambda)$ is obtained from (3), which has a unique solution for particlebased system with their monotonically increasing density of states, the canonical entropy (with Boltzmann's constant set to 1 throughout) is defined as

$$
S_{C}(E, \lambda) \equiv \beta_{C}(E, \lambda)\left[E-F\left(\beta_{C}(E, \lambda), \lambda\right)\right]=S\left(\beta_{C}(E, \lambda), \lambda\right) .
$$

Here,

$$
S(\beta, \lambda) \equiv \beta^{2} \partial_{\beta} F(\beta, \lambda)=\beta(U-F)
$$

is the standard entropy of the canonical ensemble leading to a mean energy $U=\partial_{\beta}(\beta F)$. The relation

$$
\partial_{E} S_{C}(E, \lambda)=\beta_{C}(E, \lambda)
$$

is easily verified and corresponds to the well-known thermodynamic one.

The change in canonical entropy associated with driving the system through $\lambda^{\tau}$, after final equilibration at constant $\lambda^{1}$, becomes with (5)

$$
\Delta S_{C}\left(\xi^{0}\right)=S_{C}\left(E^{0}+W\left(\xi^{0}\right), \lambda^{1}\right)-S_{C}\left(E^{0}, \lambda^{0}\right),
$$

which still depends on the initial $\xi^{0}$. Our first aim is to explore under which conditions the mean entropy change can be shown to be non-negative. It will be convenient to rewrite $\Delta S_{C}$ as

$$
\Delta S_{C}\left(\xi^{0}\right)=S_{C}\left(E^{0}+\mathcal{W}+\delta W\left(\xi^{0}\right), \lambda^{1}\right)-S_{C}\left(E^{0}, \lambda^{0}\right)
$$

with

$$
\delta W\left(\xi^{0}\right) \equiv W\left(\xi^{0}\right)-W .
$$

Here, $\mathcal{W}$ is defined as the mean work one would spend in such a process in a fictitious ensemble where the initial points are drawn from a canonical distribution at inverse temperature $\beta_{C}\left(E, \lambda^{0}\right)$. Specifically,

$$
\mathcal{W} \equiv \int d \xi^{0} \exp \left\{-\beta_{C}\left[H\left(\xi^{0}, \lambda^{0}\right)-F\left(\beta_{C}, \lambda^{0}\right)\right]\right\} W\left(\xi^{0}\right) .
$$

Expanding the first term of (9) in $\delta W\left(\xi^{0}\right)$ and using (7) leads after averaging to

$$
\left\langle\Delta S_{C}\right\rangle=\Delta \mathcal{S}+\langle\delta W\rangle \beta_{C}\left(E^{0}+\mathcal{W}, \lambda^{1}\right)+\sum_{k=2}^{\infty}(1 / k !)\left\langle\left(\delta W\left(\xi^{0}\right)\right)^{k}\right\rangle \partial_{E}^{k-1} \beta_{C}\left(E, \lambda^{1}\right)_{\mid E=E^{0}+\mathcal{W}} .
$$

Here, the leading term

$$
\Delta S \equiv S_{C}\left(E^{0}+\mathcal{W}, \lambda^{1}\right)-S_{C}\left(E^{0}, \lambda^{0}\right)
$$

corresponds to the canonical entropy change for those initial phase points $\xi^{0}$ which happen to lead to the same work as the average work in the fictitious canonical ensemble, $W\left(\xi^{0}\right)=W$. 


\subsection{A theorem}

As our first main result, we prove

$$
\Delta S \geq 0 .
$$

Consider two normalized distributions $p_{0}\left(\xi^{0}\right)$ and $p_{1}\left(\xi^{0}\right)$ that both vanish nowhere on phase space. Set $x=p_{1} / p_{0}$ in the trivial $\ln (1 / x) \geq 1-x$ and average both sides using $p_{0}$. This leads to

$$
\int d \boldsymbol{\xi}^{0} p_{0}\left(\xi^{0}\right) \ln \left[p_{0}\left(\xi^{0}\right) / p_{1}\left(\xi^{0}\right)\right] \geq 0
$$

known as positivity of the relative entropy. We choose

$$
p_{0}\left(\xi^{0}\right)=\exp \left[-\beta_{0}\left(H\left(\xi^{0}, \lambda^{0}\right)-F\left(\beta_{0}, \lambda^{0}\right)\right)\right]
$$

and

$$
p_{1}\left(\xi^{0}\right)=\exp \left[-\beta_{1}\left(H\left(\xi^{1}\left(\xi^{0}\right), \lambda^{1}\right)-F\left(\beta_{1}, \lambda^{1}\right)\right)\right]
$$

with yet arbitrary $\beta_{0}$ and $\beta_{1}$. Both distributions are normalized where for $p_{1}$ we exploit Liouville's theorem. Inserting them into the inequality 15 leads to

$$
\beta_{1}\left[E^{0}+W_{0}-F\left(\beta_{1}, \lambda^{1}\right)\right] \geq \beta_{0}\left[E^{0}-F\left(\beta_{0}, \lambda^{0}\right)\right]
$$

valid for any $\beta_{0}$ and $\beta_{1}$ with $\mathcal{W}_{0}$ the mean work associated with the driving $\lambda^{\tau}$ for an initial distribution drawn from a fictitious canonical ensemble at $\left(\beta_{0}, \lambda^{0}\right)$. Specializing to

$$
\beta_{0}=\beta_{C}\left(E^{0}, \lambda^{0}\right)
$$

which implies $\mathcal{W}_{0}=\mathcal{W}$, and to

$$
\beta_{1}=\beta_{C}\left(E^{0}+\mathcal{W}, \lambda^{1}\right)
$$

proves $\Delta S \geq 0$.

If one could replace the fluctuating work in the micro-canonical distribution by the corresponding mean canonical one, that is by $\mathcal{W}$, a second law for the entropy change were proven through the theorem. While such a replacement might seem plausible for $N \rightarrow \infty$, a more detailed analysis is warranted.

\subsection{Fluctuations in the large- $N$ limit}

We first consider a system that is non-infinitesimally driven, i.e., the control parameter changes in finite time by an order one and the driving affects a non-negligible part of the system. The sign and magnitude of the terms in the series (12) can then be estimated as follows. (i) $\Delta \mathcal{S}$ is non-negative because of the theorem. For a macroscopic system driven as just described, $\Delta S$ will typically be of the order of the number of degrees of freedom $N$. (ii) For such a process, the average of the micro-canonical work, $\left\langle W\left(\xi^{0}\right)\right\rangle$, and the mean canonical work, $\mathcal{W}$, will typically be both of order $N$. Their difference $\langle\delta W\rangle$ should then be of order one. This is most obvious for a quench, $\lambda^{0} \rightarrow \lambda^{1}$, since the work is then given by the corresponding mean values of the phase space function $H\left(\xi, \lambda^{1}\right)-E^{0}$. For such an extensive observable, canonical and micro-canonical average differ by order one as shown in the Appendix. (iii) In the sum, for $k=2,\left\langle(\delta W)^{2}\right\rangle$ will typically scale $\sim N$. Since, $\partial_{E} \beta_{C} \sim(-) 1 / N$, in the sum, the leading term with $k=2$ will be negative of order 1 .

Under these assumptions, the sum of second and third term in 12 is at most negative of order 1 . The first term, $\Delta \mathcal{S}$, is definitely non-negative and typically positive of order $N$. Contributions involving the higher order moments $\left\langle\delta W\left(\xi^{0}\right)^{k}\right\rangle, k \geq 3$ vanish for large $N$. For a macroscopic system, we have thus shown that, on the scale $N$, the canonical entropy for a driven, thermally isolated system does not decrease on average. However, the possible presence of nonextensive negative terms of $O(1)$ prevents us from proving a sharper, strict second law in the form $\left\langle\Delta S_{C}\right\rangle \geq 0$ for a finite system.

For an infinitesimal quench of order $\delta \lambda$ affecting a large system, the scaling of the first three terms in the expansion (12) with $\delta \lambda$ can be inferred as follows while their scaling with $N$ as just derived remains unchanged. The first term is 
non negative and hence will scale $\sim N(\delta \lambda)^{2}$. The second one can be of any sign and hence is linear in $\delta \lambda$. In the third one, since $\delta W \sim \delta \lambda$, we get $\left\langle(\delta W)^{2}\right\rangle \sim(\delta \lambda)^{2}$. Summarizing, for small $\delta \lambda$, we have

$$
\left\langle\Delta S_{C}\right\rangle \approx c_{1} N(\delta \lambda)^{2} \pm c_{2} \delta \lambda-c_{3}(\delta \lambda)^{2},
$$

with positive constants $c_{1,2,3}$ of order 1 . Consequently, there will generically be a one-sided small range $|\delta \lambda| \lesssim 1 / N$ for which the mean change in canonical entropy will be negative of order $-1 / N$ for a large system.

\section{Standard micro-canonical entropies}

We now turn to the status of the second law for a driven system using the two more established variants of the micro-canonical entropy, the Boltzmann entropy and the Gibbs volume entropy. The Boltzmann entropy is defined as

$$
S_{B}(E, \lambda) \equiv \ln \left[\delta E \int d \boldsymbol{\xi} \delta(E-H(\xi, \lambda))\right],
$$

where $\delta E$ is a constant parameter to render the logarithm dimensionless. It will drop out in the final expressions. The volume, or Gibbs entropy, is defined by

$$
S_{G}(E, \lambda) \equiv \ln \left[\int d \boldsymbol{\xi} \theta(E-H(\xi, \lambda))\right] .
$$

It will be convenient to define the Gibbs temperature

$$
\beta_{G}(E, \lambda) \equiv \partial_{E} S_{G}(E, \lambda)
$$

implying

$$
S_{B}=S_{G}+\ln \left(\delta E \beta_{G}\right) .
$$

We first recall the derivation of the micro-canonical Crooks relation [18] on which we will base the further analysis. The equations of motion are reversible which implies that

$$
W\left(\xi^{0}\right)=-\tilde{W}\left(\tilde{\xi}^{0}\right)
$$

where $\tilde{W}\left(\tilde{\xi}^{0}\right)$ is the work spent in the reverse process driven by the reversed protocol $\tilde{\lambda}^{\tau} \equiv \lambda^{t-\tau}$ and starting at $\tilde{\xi}^{0}$, defined as the final point $\xi^{1}\left(\xi^{0}\right)$ of the original process with reversed momenta. Consequently,

$$
\delta\left(W\left(\xi^{0}\right)-W\right)=\delta\left(\tilde{W}\left(\tilde{\xi}^{0}\right)+W\right) .
$$

Integrating this relation over the energy shell at $E^{0}$, yields the micro-canonical Crooks relation [18]

$$
\begin{aligned}
p\left(W \mid E^{0}\right) e^{S_{B}\left(E^{0}, \lambda^{0}\right)} & =\int d \xi^{0} \delta\left(H\left(\tilde{\xi}^{0}, \lambda^{0}\right)-E^{0}\right) \delta\left(\tilde{W}\left(\tilde{\boldsymbol{\xi}}^{0}\right)+W\right) \\
& =\int d \xi^{1} \delta\left(H\left(\xi^{1}, \lambda^{1}\right)-E^{0}-W\right) \delta\left(\tilde{W}\left(\tilde{\boldsymbol{\xi}}^{0}\right)+W\right) \\
& =\tilde{p}\left(-W \mid E^{0}+W\right) e^{S_{B}\left(E^{0}+W, \lambda^{1}\right)} .
\end{aligned}
$$

Here, $p(W \mid E)$ and $\tilde{p}(W \mid E)$ are the micro-canonical distributions on the energy shell $E$ for a work $W$ for the original and the reversed process, respectively. For the change in Boltzmann entropy

$$
\Delta S_{B}\left(\xi^{0}\right) \equiv S_{B}\left(E^{0}+W\left(\xi^{0}\right), \lambda^{1}\right)-S_{B}\left(E^{0}, \lambda^{0}\right),
$$

one finds, after getting $e^{S_{B}\left(E^{0}+W, \lambda^{1}\right)}$ to the left hand side and integration over $W$, the exact result

$$
\left\langle e^{-\Delta S_{B}}\right\rangle=\int d W \tilde{p}\left(-W \mid E^{0}+W\right) .
$$


Because of the conditioning on $E^{0}+W$ the integral is not 1 , in general. Consequently, one cannot infer $\left\langle\Delta S_{B}\right\rangle \geq 0$, in general, either. To proceed, we apply an expansion on the right hand side, leading to

$$
\left\langle e^{-\Delta S_{B}}\right\rangle=1+\sum_{k=1}^{\infty} \frac{(-1)^{k}}{k !} \partial_{E}^{k}\left\langle\tilde{W}^{k}\right\rangle,
$$

where $\tilde{W}$ is the work in the reversed process and the average is over the energy shell $E^{0}$. This original result is still formally exact.

We now consider a large system, for which the driving affects a substantial fraction of all degrees of freedom. We then expect $\tilde{W}$ to scale like $E$,

$$
\langle\tilde{W}\rangle \approx \tilde{\alpha} E
$$

with $\tilde{\alpha}$ of order 1 being a functional of the driving protocol. Likewise, we expect the variance of the work to be of order $E$ and a corresponding scaling of the higher cumulants. Under these assumptions the terms in the series (31) become

$$
\partial_{E}^{k}\left\langle\tilde{W}^{k}\right\rangle \approx \partial_{E}^{k}\langle\tilde{W}\rangle^{k} \approx k ! \tilde{\alpha}^{k} .
$$

Inserted in (31) and summing the geometrical series leads to the integral fluctuation relation

$$
\left\langle e^{-\Delta S_{B}}\right\rangle=1 /(1+\tilde{\alpha})
$$

for a large driven system. Jensen's inequality then implies

$$
\left\langle\Delta S_{B}\right\rangle \geq \ln (1+\tilde{\alpha}) .
$$

Since $\tilde{\alpha}$ can be negative, as exemplified below, the average change in Boltzmann entropy is, even for a large system, not necessarily positive. However, as above for the change in canonical entropy, it cannot be negative of order $E$, i.e., of $\operatorname{order} N$.

For the change in Gibbs entropy

$$
\left.\Delta S_{G}\left(\xi^{0}\right) \equiv S_{G}\left(E^{0}+W\left(\xi^{0}\right)\right), \lambda^{1}\right)-S_{G}\left(E^{0}, \lambda^{0}\right),
$$

a similar result can be derived as follows. With (25), and (28), we obtain

$$
\begin{aligned}
\left\langle e^{-\Delta S_{G}}\right\rangle & =\int d W p\left(W \mid E^{0}\right) e^{-\Delta S_{G}} \\
& =\int d W \tilde{p}\left(-W \mid E^{0}+W\right) e^{\left(\Delta S_{B}-\Delta S_{G}\right)} \\
& \left.=\frac{1}{\beta_{G}\left(E^{0}, \lambda^{0}\right)} \int d W \tilde{p}\left(-W \mid E^{0}+W\right) \beta_{G}\left(E^{0}+W\right), \lambda^{1}\right) \\
& =\frac{1}{\beta_{G}\left(E^{0}, \lambda^{0}\right)}\left[\beta_{G}\left(E^{0}, \lambda^{1}\right)+\sum_{k=1}^{\infty} \frac{(-1)^{k}}{k !} \partial_{E}^{k}\left[\left\langle\tilde{W}^{k}\right\rangle \beta_{G}\left(E, \lambda^{1}\right)\right]_{\mid E=E^{0}}\right] .
\end{aligned}
$$

Since $\beta_{G}(E, \lambda) \approx c N / E$, where $c$ is of order 1 , each term in the sum will vanish in the limit of a large system if the scaling (32) is assumed as above. Consequently, in this limit,

$$
\left\langle e^{-\Delta S_{G}}\right\rangle=\beta_{G}\left(E^{0}, \lambda^{1}\right) / \beta_{G}\left(E^{0}, \lambda^{0}\right) .
$$

The corresponding inequality becomes

$$
\left\langle\Delta S_{G}\right\rangle \geq \ln \left[\beta_{G}\left(E^{0}, \lambda^{0}\right) / \beta_{G}\left(E^{0}, \lambda^{1}\right)\right] .
$$

For a large system, the right hand side will be of order 1 but not necessarily positive. As in the two cases discussed above, we find that for a large system, the mean change in Gibbs volume entropy cannot be extensively negative, i.e., negative of order $N$. 


\section{Illustrative examples}

These results can be illustrated using exactly solvable models.

\subsection{Quenching an $N$-dimensional harmonic oscillator}

We consider an isotropic $N$-dimensional harmonic oscillator $\left(\xi \equiv\left\{q_{j}, p_{j}\right\}, j=1, \ldots, N\right)$ with mass $m$ and energy $E^{0}$ that is instantaneously quenched from an initial stiffness $\lambda^{0}$ to a final one

$$
\lambda^{1} \equiv \gamma \lambda^{0}
$$

The work spent in this process can be written as

$$
W\left(\xi^{0}\right)=(\gamma-1) x\left(\xi^{0}\right) E^{0}
$$

where

$$
x\left(\xi^{0}\right) \equiv \lambda^{0} \sum_{j=1}^{N}\left(q_{j}^{0}\right)^{2} /\left(2 E^{0}\right)
$$

denotes that fraction of the total energy which the potential energy carries initially $(0 \leq x \leq 1)$. The micro-canonical distribution $p(x)$ can be calculated exactly and is given by the symmetric beta distribution

$$
p(x)=c_{N}[x(1-x)]^{N / 2-1}
$$

with mean $\langle x\rangle=1 / 2$ and normalization $c_{N} \equiv \Gamma(N) / \Gamma^{2}(N / 2)$ involving the Gamma function.

The change in Boltzmann entropy (29) becomes

$$
\Delta S_{B}(x)=\ln \frac{[1+(\gamma-1) x]^{N-1}}{\gamma^{N / 2}} .
$$

Its mean $\left\langle\Delta S_{B}\right\rangle=\int_{0}^{1} d x p(x) \Delta S_{B}(x)$ is negative for $N=1,2$ and any $\gamma>1$. For any $N \geq 3$, there exists a range $1<\gamma<\gamma^{*}(N)$ for which $\left\langle\Delta S_{B}\right\rangle<0$. For $N \rightarrow \infty, \gamma^{*}(N) \rightarrow 1$.

The canonical entropy of the $N$-dimensional oscillator is given by

$$
S_{C}(E, \lambda)=\beta_{C}\left[E-F\left(\beta_{C}, \lambda\right)\right]=N\left[1+\ln \frac{2 \pi m^{1 / 2} E}{N h \lambda^{1 / 2}}\right]
$$

using $\beta_{C}(E, \lambda)=N / E$, and $h$ Planck's constant. Consequently, the change in canonical entropy after the quench from $\lambda^{0}$ to $\lambda^{1}$ becomes

$$
\Delta S_{C}(x)=N \ln \frac{1+(\gamma-1) x}{\gamma^{1 / 2}},
$$

which is not necessarily positive. However, the mean change $\left\langle\Delta S_{C}\right\rangle=\int_{0}^{1} d x p(x) \Delta S_{C}(x)$ is positive for any $\gamma \neq 1$ and any $N$. We also get

$$
\Delta \mathcal{S}=\Delta S_{C}(x=1 / 2)=N \ln \frac{1+\gamma}{2 \gamma^{1 / 2}} \geq\left\langle\Delta S_{C}\right\rangle \geq 0 .
$$

This result illustrates the above discussion on the sign of the third term in (12) since for this case the second term vanishes due to $W=\langle W\rangle$.

For this quench from $\lambda^{0} \rightarrow \lambda^{1}=\gamma \lambda^{0}$, the change in Gibbs volume entropy is exactly the same as in canonical entropy, $\Delta S_{G}(x)=\Delta S_{C}(x)$. Hence, the mean change in volume entropy $\left\langle\Delta S_{G}\right\rangle$ is positive for all $N$ as well. Moreover, since the Gibbs temperature is independent of the stiffness at fixed energy, as is the canonical temperature, the right hand side of (39) vanishes thus confirming the strict positivity of $\left\langle\Delta S_{G}\right\rangle$ in this case for a large system. 


\subsection{A two-dimensional harmonic oscillator quenched into a finite disc}

As a specific example for a mean negative entropy change for both the Gibbs volume entropy and the canonical one, consider a two-dimensional harmonic oscillator with microcanonical initial conditions at energy $E^{0}$. At time $t=0$, a circular wall is introduced with radius

$$
R=\left(2 E^{0} / \lambda^{0}\right)^{1 / 2}
$$

Since this is the maximal possible elongation of the oscillator from the origin, introducing this wall does not cost work. At the same time, the stiffness $\lambda^{0}$ is set to zero, thereby extracting work and thus lowering the energy of the system. For $t>0$ the system thus corresponds to a two dimensional particle confined to a circular disc with final energy

$$
E^{1}(x)=x\left(\xi^{0}\right) E^{0} .
$$

In two-dimensions, the distribution $p(x)=1$ is constant as given by (43) for $N=2$.

Initially, the Gibbs volume entropy is

$$
S_{G}\left(E^{0}\right)=\ln \left[\frac{(2 \pi)^{2}}{h^{2}} \int_{0}^{\infty} d p p \int_{0}^{\infty} d q q \theta\left(\left(E^{0}\right)^{2}-p^{2} / 2 m-\lambda^{0} q^{2} / 2\right)\right]=\ln \frac{2 \pi^{2}\left(E^{0}\right)^{2} m}{\lambda^{0} h^{2}} .
$$

Depending on the final energy [49], the final Gibbs volume entropy becomes

$$
S_{G}\left(E^{1}(x)\right)=\ln \left[\frac{(2 \pi)^{2}}{h^{2}} \int_{0}^{\infty} d p p \int_{0}^{R} d q q \theta\left(E^{1}-p^{2} / 2 m\right)\right]=\ln \frac{2 \pi^{2} E^{1}(x) m R^{2}}{h^{2}} .
$$

Consequently, the mean entropy change becomes

$$
\left\langle\Delta S_{G}(x)\right\rangle=\int_{0}^{1} d x \ln (2 x)=\ln 2-1<0 .
$$

For the canonical entropy, we have initially with $\beta_{C}=2 / E^{0}$

$$
S_{C}\left(E^{0}\right)=2+\ln \frac{(2 \pi)^{2} m\left(E^{0}\right)^{2}}{4 \lambda^{0} h^{2}} .
$$

After the quench, the particle in the two-dimensional disc has an entropy

$$
S_{C}\left(E^{1}\right)=1+\ln \frac{2 \pi^{2} m E^{1} R^{2}}{h^{2}}
$$

where we use $\beta_{C}^{1}=1 / E^{1}$. The mean entropy change thus becomes

$$
\left\langle\Delta S_{C}\right\rangle=-1+\int_{0}^{1} d x \ln (4 x)=-2+2 \ln 2<0 .
$$

For both entropy versions, the mean entropy change is thus negative in this quench. Further examples with a mean negative entropy change can be constructed [31].

\section{Concluding summary}

We have investigated whether a second law for the mean entropy change of an isolated driven system can be proven. For three variants of entropy (canonical, Boltzmann, and Gibbs volume) starting from micro-canonical initial conditions for a large system, we have shown that the average entropy change cannot be negative of order $N$. This result was based on a theorem for the canonical entropy and on explicit series expressions derived from the microcanonical Crooks relation for the two standard variants, using in all three cases rather mild assumptions on the scaling 
of the moments of the work distribution. For a large but finite system, however, the mean entropy change can very well be negative as it has been demonstrated explicitly for all three versions of entropy.

Concerning future perspectives, this study first prompts the quest for a non-existence proof of an entropy function $S(E, \lambda)$ whose mean change is non-negative for arbitrary driving $\lambda^{\tau}$ in any finite system. Since the three, arguaby most prominent, candidates of such a function have been excluded here through counter-examples, it is unlikely that any other reasonable variant will behave qualitatively differently. Second, it would be interesting to explore more generally bounds on the maximal possible negative values that can be reached for the mean entropy change in the large- $N$ limit. For infinitesimal changes of a control parameter, our series expansion for canonical entropy suggest that negative values of order $-1 / N$ are generic. The arguments based on the integral fluctuations relations for Boltzmann and Gibbs entropy given here cannot not even exclude the possibility of negative values of order 1 for finite quenches.

\section{Appendix A. N-dependence of the difference between canonical and microcanonical mean values}

In the micro-canonical ensemble of energy $E$, the mean value of an observable $A$ is given by

$$
\langle A \mid E\rangle=\int d \xi \delta(H(\xi)-E) A(\xi) \delta E e^{-S_{B}(E)},
$$

where the Boltzmann entropy

$$
S_{B}(E) \equiv\left[\ln \delta E \int d \xi \delta(H(\xi)-E)\right]
$$

with an irrelevant constant parameter $\delta E$ of dimension energy provides the normalization.

The mean value of an observable $A(\xi)$ in the canonical ensemble at inverse temperature $\beta$ and free energy $F(\beta)$ can then be expressed as

$$
\begin{aligned}
\langle A \mid \beta\rangle & =\int d \xi \exp [-\beta(H(\xi)-F(\beta))] A(\xi) \\
& =\int d E \int d \xi \exp [-\beta(H(\xi)-F(\beta))] A(\xi) \delta(H(\xi)-E) \\
& =\frac{\int d E e^{-\beta E+S_{B}(E)}\langle A \mid E\rangle}{\int d E e^{-\beta E+S_{B}(E)}},
\end{aligned}
$$

We now perform a saddle point evaluation of both integrals in $\mathrm{A.3}$ around $\hat{E}$ implicitly defined through

$$
\beta=\partial_{E} S_{B}(E)_{\mid E=\hat{E}} \equiv \beta_{B}(\hat{E}) .
$$

In the nominator, we have to expand $\langle A \mid E\rangle$ to second order and the exponent to third order in $y \equiv E-\hat{E}$. The final result is 1

$$
\langle A \mid \beta\rangle \approx\langle A \mid \hat{E}\rangle\left[1+\frac{\partial_{E}^{2}\langle A \mid E\rangle}{2\left|\partial_{E} \beta_{B}\right|\langle A \mid E\rangle}+\frac{\partial_{E}^{2} \beta_{B} \partial_{E}\langle A \mid E\rangle}{2\left|\partial_{E} \beta_{B}\right|^{2} \mid\langle A \mid E\rangle}\right]_{\mid E=\hat{E}} .
$$

The crucial point now is that in a large system $\partial_{E} \sim 1 / N$. Hence, the square bracket in A.5 is $[1+O(1 / N)]$. Consequently, the canonical and the micro-canonical average of an observable $A$ differ by a relative amount of order $1 / N$.

\footnotetext{
${ }^{1}$ For a function $f(x)$ with a narrow maximum at $\hat{x}$, hence $f^{\prime \prime}(\hat{x})<0$, a function $g(x)$, and $y \equiv x-\hat{x}$, we use

$$
\begin{aligned}
\int d x g(x) e^{f(x)} & \approx \int d y g(\hat{x})\left[1+\frac{y g^{\prime}(\hat{x}}{g(\hat{x})}+\frac{y^{2} g^{\prime \prime}(\hat{x})}{2 g(\hat{x})}\right] e^{f(\hat{x})+y^{2} f^{\prime \prime}(\hat{x})+y^{3} f^{\prime \prime \prime}(\hat{x}) / 6} \\
& \approx g(\hat{x}) e^{f(\hat{x})} \int d y\left[1+\frac{y g^{\prime}(\hat{x})}{g(\hat{x})}+\frac{y^{2} g^{\prime \prime}(\hat{x})}{2 g(\hat{x})}\right]\left[1+y^{3} f^{\prime \prime \prime}(\hat{x}) / 6\right] e^{y^{2} f^{\prime \prime}(\hat{x}) / 2} \\
& =g(\hat{x})\left[2 \pi /\left|f^{\prime \prime}(\hat{x})\right|\right]^{1 / 2}\left[1+\frac{g^{\prime \prime}(\hat{x})}{2 g(\hat{x})\left|f^{\prime \prime}(\hat{x})\right|}+\frac{g^{\prime}(\hat{x}) f^{\prime \prime \prime}(\hat{x})}{2 g(\hat{x})\left|f^{\prime \prime}(\hat{x})\right|^{2}}\right] .
\end{aligned}
$$
}




\section{Dedication}

This paper is dedicated to the memory of Christian Van den Broeck, pioneer in stochastic thermodynamics. His thought-provoking papers on the subject and the many inspiring and, reflecting his fine character, always friendly discussions we had over the last fifteen years strongly influenced my research in the field and my understanding of it.

\section{References}

\section{References}

[1] I. M. Bassett, Alternative derivation of the classical second law of thermodynamics, Phys. Rev. A 18 (1978) 2356.

[2] A. Lenard, Thermodynamical proof of the Gibbs formula for elementary quantum systems, J. Stat. Phys. 19 (1978) 575.

[3] C. Jarzynski, Nonequilibrium equality for free energy differences, Phys. Rev. Lett. 78 (1997) 2690.

[4] A. Allahverdyan, T. Nieuwenhuizen, A mathematical theorem as the basis for the second law: Thomsons formulation applied to equilibrium, Physica A 305 (2002) 542.

[5] K. Sato, An example of a mechanical system whose ensemble average energy, starting with a microcanonical ensemble, decreases after an operation, J. Phys. Soc. Japan 71 (2002) 1065.

[6] R. Marathe, J. M. R. Parrondo, Cooling classical particles with a microcanonical Szilard engine, Phys. Rev. Lett. 104 (24) (2010) 245704.

[7] R. Kawai, J. M. R. Parrondo, C. Van den Broeck, Dissipation: The phase-space perspective, Phys. Rev. Lett. 98 (2007) 080602.

[8] S. Hilbert, P. Hänggi, J. Dunkel, Thermodynamic laws in isolated systems, Phys. Rev. E 90 (2014) 062116.

[9] R. H. Swendsen, Thermodynamics, statistical mechanics and entropy, Entropy 19 (2017) 603.

[10] R. H. Swendsen, Continuity of the entropy of macroscopic quantum systems, Phys. Rev. E 92 (2015) 052110.

[11] M. Matty, L. Lancaster, W. Griffin, R. H. Swendsen, Comparison of canonical and microcanonical definitions of entropy, Physica A 467 (2017) 474

[12] J. M. G. Vilar, J. M. Rubi, Communication: System-size scaling of Boltzmann and alternate Gibbs entropies, J. Chem. Phys. 140 (2014) 201101.

[13] D. Frenkel, P. B. Warren, Gibbs, Boltzmann, and negative temperatures, Am. J. Phys. 83 (2015) 163.

[14] E. Abraham, O. Penrose, Physics of negative absolute temperatures, Phys. Rev. E 95 (2017) 012125.

[15] J. Dunkel, S. Hilbert, Consistent thermostatistics forbids negative absolute temperatures, Nature Physics 10 (2014) 67.

[16] M. Campisi, Construction of microcanonical entropy on thermodynamic pillars, Phys. Rev. E 91 (2015) 052147.

[17] R. Franzosi, Microcanonical entropy for classical systems, Physica A 494 (2018) 302.

[18] B. Cleuren, C. Van den Broeck, R. Kawai, Fluctuation and dissipation of work in a Joule experiment, Phys. Rev. Lett. 96 (2006) 050601.

[19] G. Papamikos, M. Robnik, Statistical properties of 1d time-dependent Hamiltonian systems: From the adiabatic limit to the parametrically kicked systems, J. Phys. A 44 (2011) 315102.

[20] D. Andresas, B. Batistić, M. Robnik, Statistical properties of one-dimensional parametrically kicked Hamilton systems, Phys. Rev. E 89 (2014) 062927.

[21] S.-i. Sasa, T. S. Komatsu, Thermodynamic irreversibility from high-dimensional Hamiltonian chaos, Prog. Theor. Phys 103 (2000) 1.

[22] H. Tasaki, Statistical mechanical derivation of the second law of thermodynamics, arXiv.org: cond-mat/0009206.

[23] M. Campisi, Statistical mechanical proof of the second law of thermodynamics based on volume entropy, Studies in History and Philosophy of Modern Physics 39 (2008) 181.

[24] J. M. R. Parrondo, C. Van den Broeck, R. Kawai, Entropy production and the arrow of time, New J. Phys. 11 (2009) 073008.

[25] M. Esposito, K. Lindenberg, C. Van den Broeck, New Journal of Physics 12 (2010) 013013.

[26] U. Seifert, First and second law of thermodynamics at strong coupling, Phys. Rev. Lett. 116 (2016) 020601.

[27] H. J. D. Miller, J. Anders, Entropy production and time asymmetry in the presence of strong interactions, Phys. Rev. E 95 (2017) 062123.

[28] P. Strasberg, M. Esposito, Stochastic thermodynamics in the strong coupling regime: An unambiguous approach based on coarse graining, Phys. Rev. E 95 (2017) 062101.

[29] C. Jarzynski, Stochastic and macroscopic thermodynamics of strongly coupled systems, Phys. Rev. X 7 (2017) 011008.

[30] E. Aurell, Unified picture of strong coupling stochastic thermodynamics and time reversals, Phys. Rev. E 97 (4) (2018) 042112.

[31] J. Foglszinger, Entropieproduktion für mikrokanonische Anfangsbedingungen, B.Sc. thesis, Universität Stuttgart (2018). 